\title{
PRKRA Localizes to Nuage Structures and the Ectoplasmic Specialization and Tubulobulbar Complexes in Rat and Mouse Testis
}

\author{
Junya Suzuki and Sadaki Yokota \\ Section of Functional Morphology, Division of Functional Morphology, Faculty of Pharmaceutical Sciences, \\ Nagasaki International University, Huis Ten Bosch 2825-7, Sasebo, Nagasaki 859-3298, Japan
}

Correspondence should be addressed to Sadaki Yokota; syokota123@gmail.com

Received 25 November 2013; Accepted 20 January 2014; Published 25 February 2014

Academic Editor: Carlo Pellicciari

Copyright (C) 2014 J. Suzuki and S. Yokota. This is an open access article distributed under the Creative Commons Attribution License, which permits unrestricted use, distribution, and reproduction in any medium, provided the original work is properly cited.

The cytoplasmic RNA-induced silencing complex (RISC) contains dsRNA binding proteins, including PRKRA, TRBP, and Dicer. RISC localizes to P-bodies. The nuage of the spermatogenic cells has function similar to the P-bodies. We study whether PRKRA localizes to nuage of spermatogenic cells of rat and mouse. PRKRA localized to four types of nuage structures, including aggregates of 60-90 nm particles, irregularly-shaped perinuclear granules, and intermitochondrial cement of pachytene spermatocytes, and chromatoid bodies of round spermatids. In addition, PRKRA is associated with dense material surrounding tubulobulbar complexes and with the ectoplasmic specialization. The results suggest that PRKRA functions in the nuage as an element of RNA silencing system and plays unknown role in the ectoplasmic specialization and at the tubulobulbar complexes of Sertoli cells attaching the head of late spermatids.

\section{Introduction}

PRKRA, a protein kinase, interferon-inducible double stranded RNA dependent activator protein kinase, also known as PACT, a protein activator of the interferon-induced protein kinase, is a product of PRKRA gene $[1,2]$ and is a member of the dsRNA binding protein family [3]. PRKRA is contained in the cytoplasmic RNA-induced silencing complex (RISC) together with transactivation response RNA binding protein (TRBP) and Dicer [4-6]. These proteins process premicroRNAs into mature microRNAs (miRNAs) that target specific mRNA species for regulation. RISC assembles into processing bodies (P-bodies) which are specific cytoplasmic foci in somatic eukaryotic cells [7-9]. P-bodies consist of many enzymes involved in mRNA turnover and play fundamental roles in mRNA decay $[10,11]$, microRNA mediated mRNA silencing [12], decapping and degradation of mRNA $[8,9,11,13]$, and storage of mRNA $[11,14]$. On the other hand, there are specific cytoplasmic foci similar to the P-bodies in spermatogenic cells, which are called nuage [15-18].
Russell and Frank [19] classified the nuage of spermatogenic cells into six different types according to its morphology and relationship with other surrounding organelles. The application of IEM to the research of nuage was first performed by Biggiogera et al. [20], followed by Moussa et al. [21]. From these studies a new step of nuage research was started. Previously, we investigated the localization of several nuage-resident proteins in the nuage structures of spermatogenic cells by immunoelectron microscopy (IEM) [22-26]. On the basis of localization of nuage proteins, including DDX4, DDX25, BRUNOL2, NANOS1, and MAEL, we reclassified the nuage into (1) aggregates of 60-90 nm particles (6090P); (2) irregularly-shaped perinuclear granules (ISPG); (3) intermitochondrial cement (IMC); (4) satellite bodies (SB); and (5) chromatoid bodies (CB) [25]. Cluster of $30 \mathrm{~nm}$ particles which were classified into the nuage by Russell and Frank [19] were not nuage because they did not contain any nuage proteins. In addition, we determined that several nuage proteins localize to spermatogenic cell-specific structures, including mitochondria-associated granules (MAG), 
granulated bodies (GB), reticulated bodies (RB), and ribosome aggregates (RA) [22-26]. These structures first appear in the cytoplasmic lobe of elongated step 10 spermatids and subsequently disappear in step 19 spermatids. While their morphology and relationship with the other organelles have been described in detail [27], their function is quite unknown.

PRKRA is contained in RISC together with Dicer, Ago2, and TRBP [5]. The RISC assembles into P-bodies. It is known that the nuage resembles the P-bodies in its function [16]. These facts suggest that PRKRA is a constituent protein of the nuage. However, it is unclear whether PRKRA is localized in the nuage structures classified into five types and also in the nonnuage structures. In addition, it is not known when PRKRA appears in and disappears from the nuage structures and the nonnuage structures during spermatogenesis. In the present study, we investigated the localization of PRKRA in the mammalian testis by immunofluorescence and immunoelectron microscopic techniques.

\section{Materials and Methods}

2.1. Animals. Male Wistar rats (240-300 g in body weight) and mice $(25 \mathrm{~g}$ ) were purchased from Kyudo Co. Ltd. (Tosu, Japan) and fed appropriate standard diets and water ad libitum until use. All experiments were performed in accordance with the Guidance for Animal Experiments issued by the Nagasaki International University.

2.2. Antibodies and Related Probes. Mouse PRKRA monoclonal antibody (M01), clone 1B9-1A7, was purchased from Abnova. Rabbit anti-DDX4 (VASA) was used as described previously by Onohara et al. [22]. Alexa 568 or Alexa 488 conjugated goat anti-rabbit IgG and goat anti-mouse IgG were purchased from Molecular Probes (Eugene, OR, USA). Horseradish peroxidase (HRP)-labeled goat antibodies to mouse IgG was purchased from DAKO Japan (Tokyo, Japan). Protein A/G/L-gold (15-nm) probes were prepared as described previously [28].

2.3. Western Blotting. Testes of rat and mice were homogenized in $5 \mathrm{mM}$ MOPS-KOH buffer ( $\mathrm{pH}$ 7.4) containing $0.25 \mathrm{M}$ sucrose, $1 \mathrm{mM}$ ethylenediaminetetraacetic acid, $1 \mathrm{mM}$ phenylmethylsulfonyl fluoride, and $1 \mu \mathrm{g} / \mathrm{mL}$ each of leupeptin, pepstatin, aprotinin, and antipain (medium A), using a Potter-Elvehjem homogenizer. Ten percent homogenates $(\mathrm{w} / \mathrm{v})$ were centrifuged at $800 \times \mathrm{g}$ for $10 \mathrm{~min}$ and the resulting supernatants were centrifuged at $10,000 \times \mathrm{g}$ for $20 \mathrm{~min}$. The resulting pellets, which were designated as mitochondrial fraction, were suspended in a minimal volume of medium $A$ and centrifuged. The mitochondria-free supernatants were centrifuged at $100,000 \times \mathrm{g}$ for $60 \mathrm{~min}$ in a Beckman ultracentrifuge equipped with a SW 41 swinging bucket rotor. The resulting pellets were suspended in medium A and designated as the microsomal fractions, while the supernatants were designated as the cytosolic fraction. The cell fractions were stored at $-70^{\circ} \mathrm{C}$. Protein concentrations were determined by the bicinchoninic acid method (Pierce Chemical, Rockford, IL) using bovine serum albumin (BSA) as a standard. The protein concentrations of the fractions were adjusted to $1 \mathrm{mg} / \mathrm{mL}$, combined with one volume of sample buffer for SDS-PAGE, and heated in a boiling water bath for $5 \mathrm{~min}$. Ten micrograms of each sample was analyzed by Western blotting. The apparent molecular mass of PRKRA was estimated by protein makers (TAKARA BIO, Tokyo, Japan).

2.4. Immunofluorescence (IF) Staining of Testis Tissue Sections. Rat and mice testes were embedded in Tissue-Tek optimal cutting temperature compound (SAKURA Finetec, Tokyo, Japan) and frozen in liquid nitrogen-cooled isopentane. Frozen sections were cut at a thickness of $6 \mu \mathrm{m}$ with a Leitz cryostat (Leica Instruments, Nussloch, Germany) and picked up on silicone-coated glass slides. Sections were fixed in $0.1 \mathrm{M}$ HEPES-KOH buffer ( $\mathrm{pH} 7.4$ ) containing 4\% paraformaldehyde (w/v), $0.01 \% \mathrm{CaCl}_{2}(\mathrm{w} / \mathrm{v})$, for $15 \mathrm{~min}$ at room temperature (RT). Sections were then treated with $0.2 \%$ Triton X$100(\mathrm{v} / \mathrm{v})$ and $0.2 \%$ Saponin (w/v) for $15 \mathrm{~min}$ and incubated in blocking solution containing $2 \%$ fish gelatin $(\mathrm{w} / \mathrm{v})$ and $15 \mathrm{mM} \mathrm{NaN}_{3}$ in PBS for $30 \mathrm{~min}$. The sections were subsequently incubated with mouse PRKRA monoclonal antibody $(5 \mu \mathrm{g} / \mathrm{mL})$ overnight at $4^{\circ} \mathrm{C}$. After washing in PBS, sections were incubated with Alexa 488- or Alexa 568-labeled goat anti-mouse IgG solutions containing $3 \mu \mathrm{M}$ DAPI (Hoechst, Tokyo, Japan) for $60 \mathrm{~min}$ at RT. For the control, incubation with serum from nonimmunized mouse was used as the primary antibody, followed by Alexa 488- or Alexa 568labeled secondary antibodies. For dual staining, sections were incubated with mixtures of rabbit anti-DDX4/mouse antiPRKRA, followed by incubation with Alexa 488- or 568labeled secondary antibodies. The sections were examined with a Nikon Eclipse E600 fluorescence microscope (Nikon, Tokyo, Japan). The images were merged using Adobe Photoshop 7.0 to determine whether each antigen colocalizes to the same area. The stage of seminiferous cycle was determined from the localization of elongating and elongated spermatids with individual tubules as previously described [29].

2.5. Immunoelectron Microscopy (IEM). Rat and mice testes were cut into small tissue blocks in $0.05 \mathrm{M}$ Hepes- $\mathrm{KOH}$ buffer ( $\mathrm{pH} 7.4$ ) containing $4 \%$ paraformaldehyde (w/v), $0.2 \%$ glutaraldehyde $(\mathrm{v} / \mathrm{v})$, and $0.02 \% \mathrm{CaCl}_{2}(\mathrm{w} / \mathrm{v})$ and fixed in the same buffer for $1 \mathrm{~h}$ at $4^{\circ} \mathrm{C}$. After washing in PBS, fixed tissue blocks were dehydrated in ethanol and embedded in LR White at $-20^{\circ} \mathrm{C}$, followed by resin polymerization under UV light at $-20^{\circ} \mathrm{C}$. Thin sections were cut with a Reichert Ultracut $\mathrm{R}$, mounted onto nickel grids, and stored in a desiccator. Sections were treated with $2 \%$ fish gelatin in PBS (v/v) and incubated with mouse PRKRA antibody $(5 \mu \mathrm{g} / \mathrm{mL})$ overnight at $4^{\circ} \mathrm{C}$. Sections were then incubated with protein $\mathrm{A} / \mathrm{G} / \mathrm{L}-$ gold probe $(15 \mathrm{~nm})$. For control, serum from nonimmunized mouse was used as the primary antibody. Sections were contrasted with uranyl acetate and lead citrate and examined with a Hitachi H7650 electron microscope (Tokyo, Japan) at an acceleration voltage of $80 \mathrm{kV}$. The stage of seminiferous cycle and step of spermatids were determined as described by Russell et al. [29].

2.6. Stage-Specific Localization of PRKRA during Spermatogenesis. Stage-specific localization of PRKRA in the nuage 


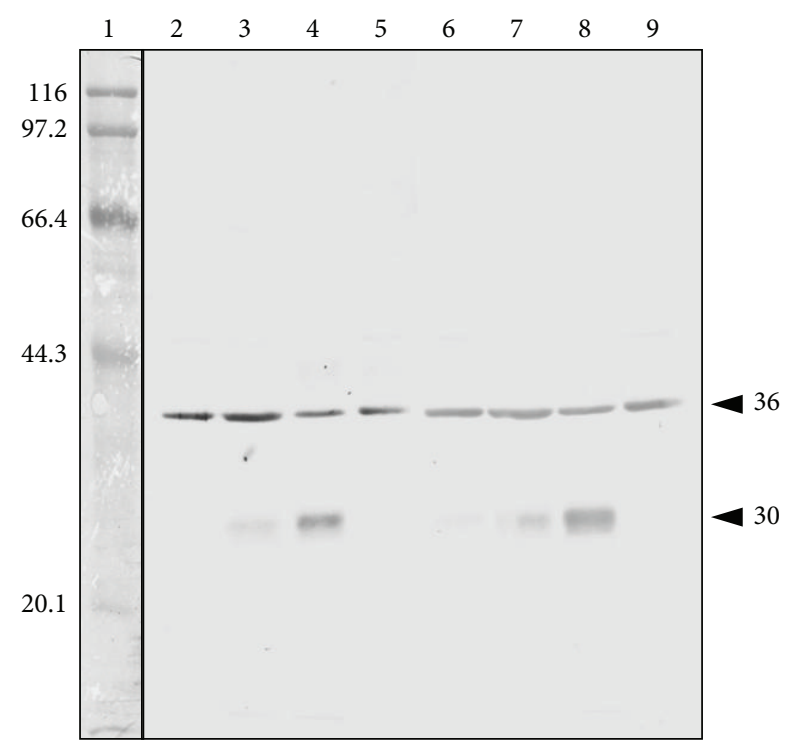

FIGURE 1: Western blotting analysis of cell fractions isolated from rat and mouse testis homogenates. Lane 1, molecular markers; lanes 2-5, rat testis; lanes 6-9, mouse testis; lanes 2 and 6, homogenates; lanes 3 and 7, mitochondrial fractions; lanes 4 and 8, microsomal fractions; lanes 5 and 9, cytosolic fractions. In the homogenates and cytosolic fractions, a single band of $36 \mathrm{kDa}$ was observed, whereas in the mitochondrial and microsomal fractions two bands of 36 and $30 \mathrm{kDa}$ were noted.

structures and cytoplasm of spermatogenic cells and Sertoli cells of rat testis was followed during spermatogenesis integrating IF and IEM staining data. The results were illustrated in a table modified from the table showing stages of the cycle of the seminiferous epithelium by Russell et al. [29].

\section{Results}

3.1. Western Blotting. A single band of $36 \mathrm{kDa}$ was observed in testis homogenates of rat and mouse with the mouse PRKRA monoclonal antibody (Figure 1). In the mitochondria and microsomal fractions isolated from rat and mouse testis homogenates, two bands of 36 and $30 \mathrm{kDa}$ were observed (Figure 1). The $36 \mathrm{kDa}$ protein appeared in all cell fractions, whereas the $30 \mathrm{kDa}$ protein was detected in mitochondria and microsomal fractions but not in cytosolic fraction. The $36 \mathrm{kDa}$ and $30 \mathrm{kDa}$ proteins were approximately consistent with the molecular masses of PRKRA isoform 1 and isoform 3 reported in Swiss-Prot, respectively. The $36 \mathrm{kDa}$ protein was contained in all cell fractions with the same amount but the $30 \mathrm{kDa}$ protein was contained the most in the microsomal fraction. Consequently, two different-sized proteins were detected in rat and mouse testis with mouse anti-PRKRA antibody.

3.2. Immunofluorescence (IF) Staining. PRKRA staining patterns of rat and mouse were similar. PRKRA was stained in the cytoplasm and discrete granules of all spermatogenic cells and Sertoli cells. First we focused on the localization of
PRKRA in differentiating spermatogenic cells. In spermatogonia, the cytoplasm was diffusely stained for PRKRA. In pachytene spermatocytes at stage I-IV, weak and diffuse staining was observed in the cytoplasm (Figure 2(a)) and small granular staining became visible as stage progressed (Figures 2(e) and 2(i)). Dual staining of PRKRA and DDX4, a marker protein for nuage, showed that these granules were also positive for DDX4 (Figures 2(e)-2(g), 2(i)-2(k)). After stage XI, the small granules decreased and instead large irregularly-shaped granules were visible (Figure $2(\mathrm{~m})$ ). Some of these granules were stained only for PRKRA (Figure 2(o), circles), but the others were positive for both the proteins. In addition, DDX4-positive small granules were located near or attached to the only PRKRA-positive granules (Figure 2(o), circles), suggesting that the small and large granules are dynamically repeating fusion and fission with each other.

Next we observed spermatids. In step 1-6 spermatids, large granules were stained for PRKRA (Figures 2(a) and 2(e)). By dual staining of PRKRA and DDX4 these granules were also positive for DDX4 (Figures 2(c) and 2(g)). In spermatids after step 7 , weak diffuse staining was observed in the cytoplasm but no granular staining was noted (Figures $2(\mathrm{i})$ and $2(\mathrm{~m}))$. Instead, diffuse staining appeared around the head of spermatids. This staining was visible in the region surrounding the acrosome of step 10 spermatids and expanded to whole head and finally wrapped the elongated cytoplasmic lobe of late spermatids (Figures 2(a), 2(e), 2(i), and $2(\mathrm{~m})$, arrows). Frequently, this staining continued to the basal region of seminiferous tubules (Figure 2(a)). DDX4 staining did not overlap with these staining patterns (Figures 2(c), 2(g), 2(k), and 2(o)), which suggest that PRKRA stained the cytoplasm of Sertoli cells. No staining was observed in control sections (Figures 2(d), 2(h), 2(l), and 2(p)).

3.3. Immunoelectron Microscopy (IEM). Gold particles showing PRKRA were observed in the nuage structures, including aggregates of $60-90 \mathrm{~nm}$ particles (60-90P), irregularlyshaped perinuclear granules (ISPG), intermitochondrial cement (IMC) of middle and late pachytene spermatocytes, and chromatoid bodies $(\mathrm{CB})$ of round spermatids (Figures 3(a)-3(f)). PRKRA associated with dense material comprised these structures. Late CB observed in steps $8-11$ spermatids were not stained for PRKRA. Another nuage structure, satellite body was negative for PRKRA. The labeling intensity and pattern were similar in rat and mouse. Nonnuage structures in which nuage proteins had been found [22-26] were not stained for PRKRA. In control sections, no gold labeling was observed (Figures 3(i)-3(l)).

Next, we observed the region surrounding the heads of late spermatids of rat, which was stained strongly for PRKRA by IF. In high magnification of IF images, diffuse and punctate staining for PRKRA was observed in the cytoplasm of Sertoli cells facing the ventral and dorsal surface of sickleshaped spermatid head (Figure 4(a)). The punctate staining was in close contact with the nuclear surface of spermatids (Figure 4(a), arrows and arrowheads). By IEM, PRKRA was closely associated with dense materials located in the ectoplasmic specialization surrounding the head (Figure 4(b), 


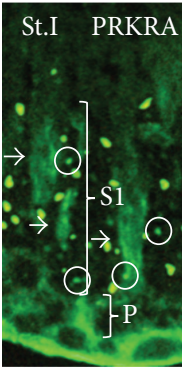

(a)

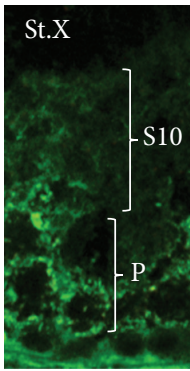

(i)

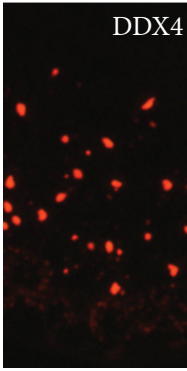

(b)

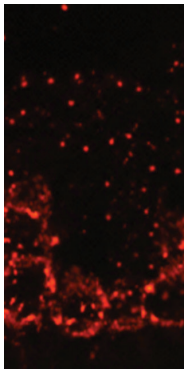

(j)

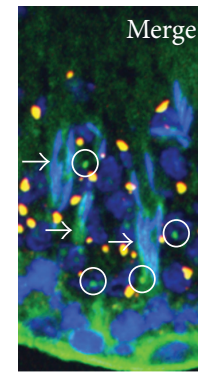

(c)

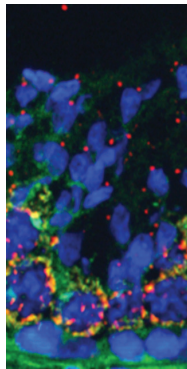

(k)

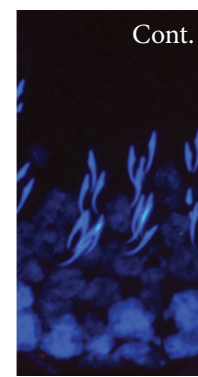

(d)

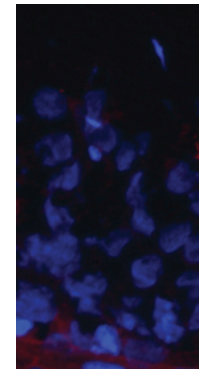

(1)

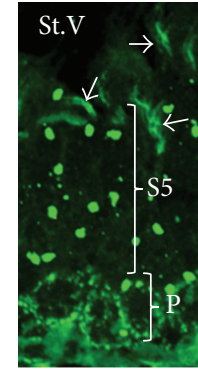

(e)

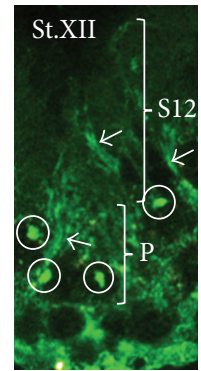

(m)

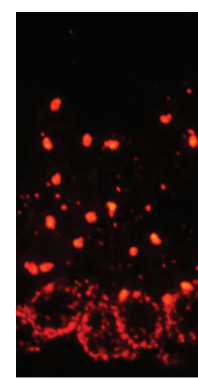

(f)

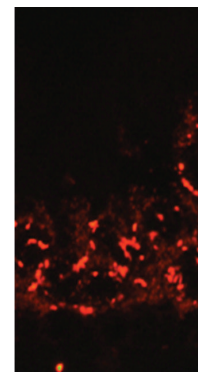

(n)

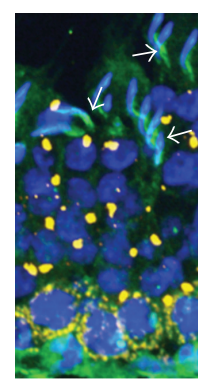

(g)

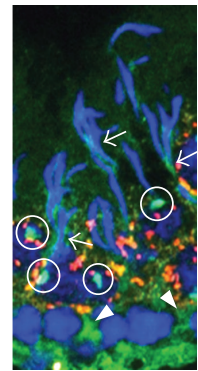

(o)

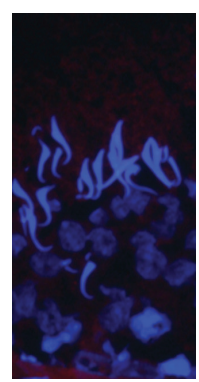

(h)

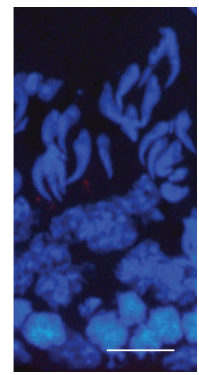

(p)

FIGURE 2: Dual staining of PRKRA (green) and DDX4 (red) in seminiferous tubules from rat. (a-d) Stage I. The cytoplasm of pachytene spermatocytes (P) was diffusely stained for PRKRA but not for DDX4. In step 1 spermatids (S1), large granules were positive for both PRKRA and DDX4 (c) but small granules were positive only for PRKRA (circles). Regions surrounding the heads of step 15 spermatids were stained for PRKRA (arrows). In control section, no PRKRA staining was observed (d). (e-h) Stage V. Small granules in pachytene spermatocytes (P) were stained for both PRKRA and DDX4. Large granules in step 5 spermatids (S5) were positive for both proteins. PRKRA was stained in region adjacent to the acrosome of step 17 spermatids (arrows). No PRKRA staining was noted in control section (h). (i-l) Stage X. Small irregularlyshaped perinuclear granules in pachytene spermatocytes $(\mathrm{P})$ were positive for both PRKRA and DDX4. The cytoplasm of step 10 spermatids (S10) was diffusely stained for PRKRA, whereas DDX4 stained small granules which were closely in contact with the nucleus (k). No PRKRA staining was seen in control (l). (m-p) Diffuse and granular staining of PRKRA was observed in pachytene spermatocytes (P). Many of these granules were also stained for DDX4-positive granules, but some were not (circles). These DDX4-positive granules were located near or attached to the PRKRA-positive granules (circles). PRKRA stained strongly regions surrounding the heads of step 12 spermatids (arrows) and diffusely the cytoplasm. The cytoplasm of zygotene spermatocytes was stained for PRKRA (arrows). In control section, no PRKRA staining was observed. Bar $=50 \mu \mathrm{m}$.

arrows). Furthermore, in the Sertoli cell cytoplasm at the ventral surface, dense materials surrounding tubulobulbar complexes were labeled for PRKRA (Figure 4(b), arrowheads). Vacuoles in the cytoplasm of Sertoli cells were negative for PRKRA (Figure 4(b), *). In control sections, no gold labeling was noted (Figure $4(\mathrm{c})$ ). In mouse testis, similar staining was observed (data not shown).

\subsection{Stage-Specific Localization of PRKRA in Rat Testis during} Spermatogenesis. The results are shown in Figure 5. PRKRApositive nuage structures, including 60-90P, ISPG, and IMC, became visible in pachytene spermatocytes at stage $\mathrm{V}$ and disappeared at stage XIII. Nuage stained for only PRKRA were observed in pachytene spermatocytes during stages from XII to XIII. PRKRA-positive CBs were detected in spermatids during steps from 1 to 6 . Late CBs present in spermatids after step 7 were not stained for PRKRA. Cytoplasmic staining in spermatocytes was weak but visible during all stages and that in spermatids was discernible during steps from 1 to 18. Cytoplasmic staining in Sertoli cells, which surrounded spermatid head, appeared in the vicinity of step 9 spermatids and was maintained until releasing of sperm.

\section{Discussion}

4.1. PRKRA Isoform 3 Is Contained Mainly in the Microsomal Fraction. Our Western blotting analysis showed that two proteins of 36 and $30 \mathrm{kDa}$ were detected in cell fractions isolated from rat and mouse testis homogenates. The $36 \mathrm{kDa}$ protein was present in all cell fractions but the $30 \mathrm{kDa}$ protein was contained predominantly in the microsomal fraction. According to database (Swiss-Prot), the $36 \mathrm{kDa}$ protein and $30 \mathrm{kDa}$ are PRKRA isoform 1 and isoform 3, respectively. The amino acid sequence of the isoform 3 is identical with that of the isoform 1 from which the $\mathrm{N}$-terminal 25 amino acids are deleted. The anti-PRKRA monoclonal antibody used is raised to a full-length recombinant PRKRA (datasheet of manufacturer). The present results strongly suggest that the antibody can recognize somewhere the sequence after but not before 26th amino acid at the N-terminus, because the antibody specific for 25 amino acids sequence at the $\mathrm{N}$-terminus of the isoform 1 cannot recognize the isoform 3. In addition, our study showed that the PRKRA isoform 3 was not contained in the cytosolic fraction. This means that the isoform does not exist as soluble form but particulate-bound form. Since it is 


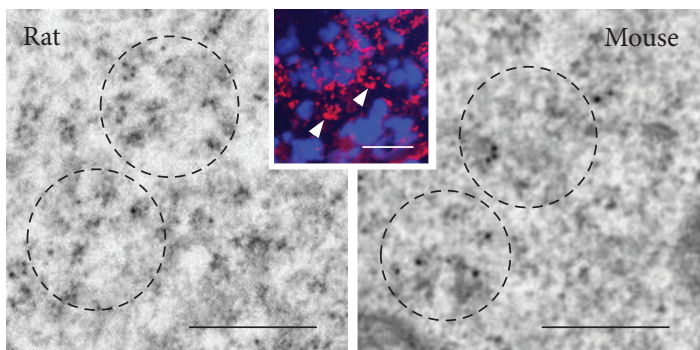

(a)

(b)

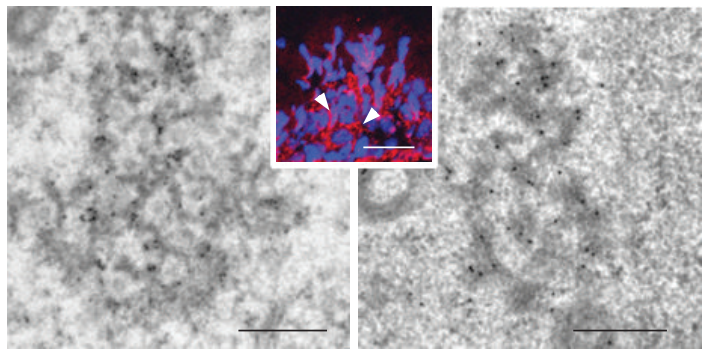

(c)

(d)

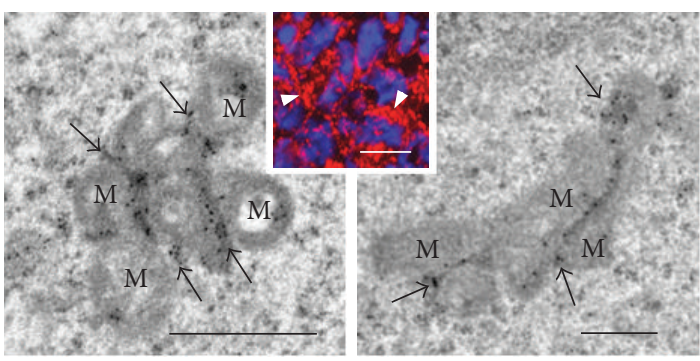

(e)

(f)

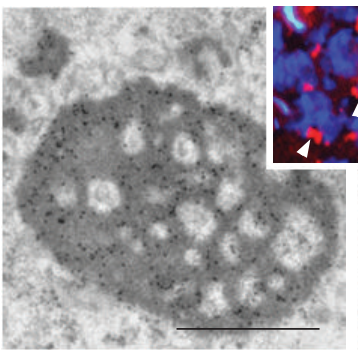

(g)

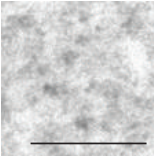

(i)

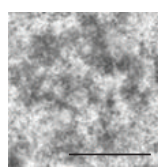

(j)

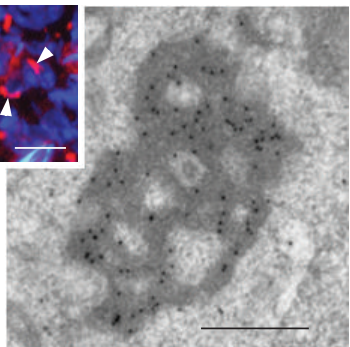

(h)

FIgURE 3: Localization of PRKRA in nuage structures by IEM. Gold particles indicate PRKRA antigenic sites. (a, c, e, and g) Samples from rat. (b, d, f, and h) Samples from mouse. All insets show IF images corresponding to IEMimages (arrowheads). (a, b) Aggregates of 60-90 nm particles (60-90P) were labeled with gold particles (circles). (a) A rat pachytene spermatocyte at stage X. (b) A mouse pachytene spermatocyte at stage IX. (c, d) PRKRA stainedirregularly-shaped perinuclear granules (ISPG). (c) A rat pachytene spermatocyte at stage IX. (d) A mouse pachytene spermatocyte at stage IX. (e, f) PRKRA localized to intermitochondrial cement (IMC) between mitochondria (M) (arrows). (e) A rat spermatocyte at stage X. (f) A mouse spermatocyte at stage IX. (g, h) Chromatoid bodies (CB) were stained for PRKRA. (g) A rat step 5 spermatids. (h) A mouse step 4 spermatid. (i) Control for 60-90P. (j) Control for ISPG. (k) Control for IMC. (l) Control for CB. Bar $=25 \mu \mathrm{m}$ for insets, $0.5 \mu \mathrm{m}$ for all IEM images. 


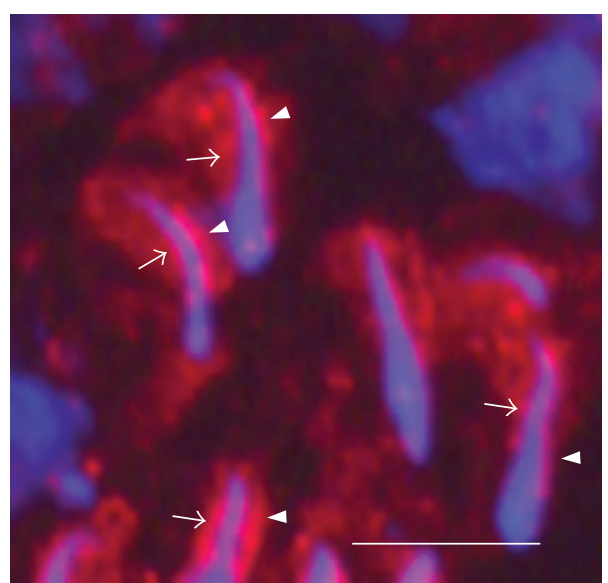

(a)

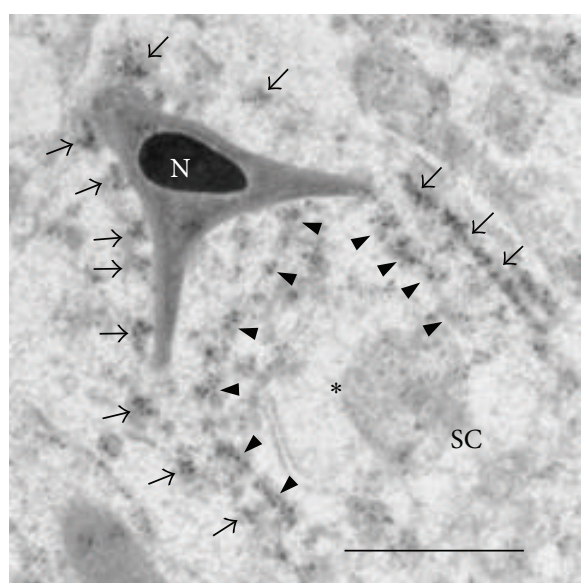

(b)

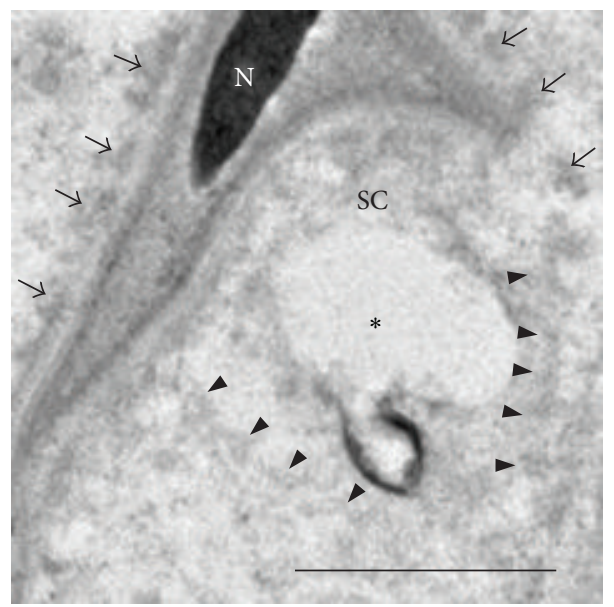

(c)

FIGURE 4: PRKRA staining in regions surrounding the sickle-shaped heads of late spermatids of rat. (a) An IF image. Strong punctate staining was observed at both the ventral (arrows) and dorsal (arrowheads) sides of sickle-shaped heads. (b) IEM staining of PRKRA. Gold labeling associated with dense material in the ectoplasmic specialization of Sertoli cell (arrows) and also with dense material attached to the tubulobulbar complexes in the Sertoli cell cytoplasm (SC) (arrowheads). A vacuole in the Sertoli cell was negative for PRKRA (*). (c) Control. Note that no gold labeling was observed in the dense material at the ectoplasmic specialization (arrows), that attached to the tubulobulbar complexes, and the vacuole $(*)$. N, nucleus. Bar $=10 \mu \mathrm{m}$ for (a) and $=1 \mu \mathrm{m}$ for (b) and (c).

likely that 60-90P and ISPG, which are positive for PRKRA, coprecipitated with the microsomal fraction and partly the mitochondrial fraction too, the isoform 3 is a constituent of the 60-90P and ISPG. If antibody specific to 25 amino acid residues at the $\mathrm{N}$-terminus of the isoform 1 could be used, $\mathrm{CB}$ and IMC were stained but the 60-90P and ISPG might not.

4.2. PRKRA Associated with Nuage Structures, including 6090P, ISPG, IMC, and CB. In the present study, dual IF staining of PRKRA and DDX4 as well as IEM labeling of PRKRA showed that PRKRA localized to five types of nuage structures, including 60-90P, ISPG, IMC, and CB. PRKRApositive $\mathrm{CB}$ was observed only in step 1-6 spermatids but not in step 7-11 ones. Previously, we showed that although the staining intensity decreased in the elongated spermatids, the nuage-resident proteins, such as DDX4, DDX25, BRUNOL2, NANOS1, and MAEL, were detected in all CB including late $\mathrm{CB}$ maintained until step 11 [22-26]. The results suggest that PRKRA function in the $\mathrm{CB}$ is restricted to the round spermatids until step 7, unlike the other nuage proteins. Our dual IF staining of PRKRA and DDX4 demonstrated that ISPG in pachytene spermatocytes at stage XII exhibited various staining patterns: staining for only PRKRA, for both proteins, for only DDX4, and chimeric staining for both proteins. Moreover, small granules positive for only DDX4, which were identified as aggregates of 60-90P by IEM, were located nearby or attached to DDX4-negative ISPG. These results suggest that in late pachytene spermatocytes, nuage structures dynamically might repeat fission and fusion with each other.

PRKRA, Ago2, Dicer, and TRBP interact with one another and form the RISC where Ago2 cleaves the target mRNA strand complementary to their bound siRNA generated from double-stranded RNAs (dsRNAs) by Dicer [6, 30, 31]. The RNA interference (RNAi) pathway plays important role in the control of transposons in the male germline $[32,33]$. 


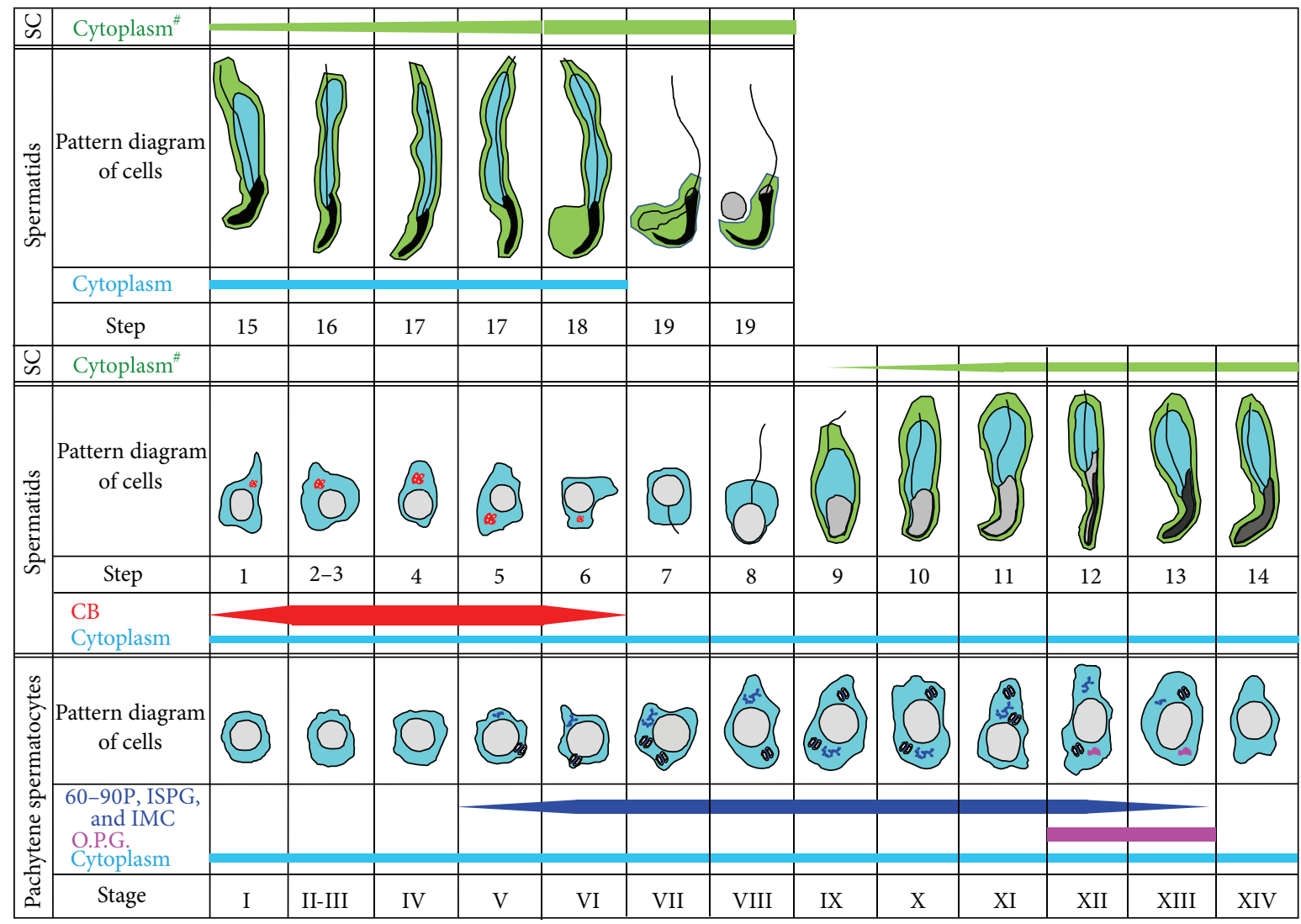

Explanation of symbols
: ISPG

FIGURE 5: Stage-specific localization of PRKRA in the nuage and the cytoplasm of spermatogenic cells and Sertoli cells during spermatogenesis of rat testis. Width of colored bars indicates staining intensity estimated arbitrarily. Appearances of ISPG and IMC are indicated by blue color, CB by red, only PRKRA-positive granules (O.P.G.) by pink, cytoplasm by cyan blue, and cytoplasm of Sertoli cells (SC), green. Early spermatocytes, including leptotene and zygotene spermatocytes, were not estimated. ${ }^{\#}$ The estimation was confined to the staining in region surrounding the sick-shaped heads of late spermatids. Illustration showing developing cells is modified from Russell et al. [27].

The present study suggests that in the spermatogenic cells, intracellular sites for this pathway are 60-90P, ISPG, and IMC in the pachytene spermatocytes and $\mathrm{CB}$ in the round spermatids.

\subsection{PRKRA Localizes to the Dense Materials at the Ectoplasmic} Specialization and Tubulobulbar Complexes in the Cytoplasm of Sertoli Cell. The ectoplasmic specialization is a subsurface modification of the Sertoli cell, composed of subsurface bundles of actin filaments and more deeply placed endoplasmic reticulum, and is a cell-cell actin-based anchoring junction [34-36]. The present study showed that PRKRA localized to the ectoplasmic specialization. Since no nuage-resident proteins have been detected in the ectoplasmic specialization so far [22-26], it is unlikely that PRKRA exerts its original function such as the RNA interference. The role of PRKRA in the ectoplasmic specialization is quite unclear.

The tubulobulbar complexes project from the ventral concavity of the head to indent the Sertoli cell, which are composed of tubules and bulbus components. When the complexes were discovered, it was considered that they were an anchoring device between Sertoli cells and late spermatids [37], but later they possibly were concerned in the elimination of the head cytoplasm of late spermatids [38]. However, the authentic function in spermatogenesis is almost not known. Although in the present study PRKRA was detected in dense materials surrounding the tubulobulbar complexes, it is unlikely that PRKRA works in the RNA interference, because no other nuage proteins have been found there so far. The functional relationship between PRKRA and tubulobulbar complexes is completely unknown. The role of PRKRA in the ectoplasmic specialization and the tubulobulbar complexes is future problem to be solved.

\section{Conflict of Interests}

The authors declare that there is no conflict of interests regarding the publication of this paper. 


\section{Acknowledgments}

The work was supported by the University research fund, in part by a Grant-in-Aid (17570158) from the Ministry of Education, Science, Culture and Sport, and by the Science Research Promotion Fund from the Promotion and Mutual Aid Corporation for Private Schools of Japan.

\section{References}

[1] R. C. Patel and G. C. Sen, "PACT, a protein activator of the interferon-induced protein kinase, PKR," The EMBO Journal, vol. 17, no. 15, pp. 4379-4390, 1998.

[2] T. Ito, M. Yang, and W. S. May, "RAX, a cellular activator for double-stranded RNA-dependent protein kinase during stress signaling," The Journal of Biological Chemistry, vol. 274, no. 22, pp. 15427-15432, 1999.

[3] L. R. Saunders and G. N. Barber, "The dsRNA binding protein family: critical roles, diverse cellular functions," FASEB Journal, vol. 17, no. 9, pp. 961-983, 2003.

[4] A. D. Redfern, S. M. Colley, D. J. Beveridge et al., "RNA-induced silencing complex (RISC) Proteins PACT, TRBP, and Dicer are SRA binding nuclear receptor coregulators," Proceedings of the National Academy of Sciences of the United States of America, vol. 110, no. 16, pp. 6536-6541, 2013.

[5] M. Sand, M. Skrygan, D. Georgas et al., "Expression levels of the microRNA maturing microprocessor complex component DGCR8 and the RNA-induced silencing complex (RISC) components argonaute-1, argonaute-2, PACT, TARBP1, and TARBP2 in epithelial skin cancer," Molecular Carcinogenesis, vol. 51, no. 11, pp. 916-922, 2012.

[6] Y. Lee, I. Hur, S.-Y. Park, Y.-K. Kim, M. R. Suh, and V. N. Kim, "The role of PACT in the RNA silencing pathway," The EMBO Journal, vol. 25, no. 3, pp. 522-532, 2006.

[7] P. Anderson and N. Kedersha, "RNA granules," Journal of Cell Biology, vol. 172, no. 6, pp. 803-808, 2006.

[8] R. Parker and U. Sheth, "P bodies and the control of mRNA translation and degradation," Molecular Cell, vol. 25, no. 5, pp. 635-646, 2007.

[9] T. M. Franks and J. Lykke-Andersen, "The control of mRNA decapping and P-body formation," Molecular Cell, vol. 32, no. 5, pp. 605-615, 2008.

[10] M. A. Ferraiuolo, S. Basak, J. Dostie, E. L. Murray, D. R. Schoenberg, and N. Sonenberg, "A role for the eIF4E-binding protein 4E-T in P-body formation and mRNA decay," Journal of Cell Biology, vol. 170, no. 6, pp. 913-924, 2005.

[11] U. Sheth and R. Parker, "Decapping and decay of messenger RNA occur in cytoplasmic processing bodies," Science, vol. 300, no. 5620, pp. 805-808, 2003.

[12] S.-P. Chan and F. J. Slack, "microRNA-mediated silencing inside P-bodies,” RNA Biology, vol. 3, no. 3, pp. 97-100, 2006.

[13] M. A. Valencia-Sanchez, J. Liu, G. J. Hannon, and R. Parker, "Control of translation and mRNA degradation by miRNAs and siRNAs," Genes and Development, vol. 20, no. 5, pp. 515-524, 2006.

[14] S. A. Barbee, P. S. Estes, A. Cziko et al., "Staufen- and FMRPcontaining neuronal RNPs are structurally and functionally related to somatic P bodies," Neuron, vol. 52, no. 6, pp. 997-1009, 2006.

[15] N. Kotaja, S. N. Bhattacharyya, L. Jaskiewicz et al., "The chromatoid body of male germ cells: similarity with processing bodies and presence of dicer and microRNA pathway components,"
Proceedings of the National Academy of Sciences of the United States of America, vol. 103, no. 8, pp. 2647-2652, 2006.

[16] N. Kotaja and P. Sassone-Corsi, "The chromatoid body: a germcell-specific RNA-processing centre," Nature Reviews Molecular Cell Biology, vol. 8, no. 1, pp. 85-90, 2007.

[17] O. Meikar, M. Da Ros, H. Korhonen, and N. Kotaja, "Chromatoid body and small RNAs in male germ cells," Reproduction, vol. 142, no. 2, pp. 195-209, 2011.

[18] E. Voronina, G. Seydoux, P. Sassone-Corsi, and I. Nagamori, "RNA granules in germ cells," Cold Spring Harbor Perspective of Biology, vol. 3, no. 12, Article ID a002774, 2011.

[19] L. Russell and B. Frank, "Ultrastructural characterization of nuage in spermatocytes of the rat testis," Anatomical Record, vol. 190, no. 1, pp. 79-98, 1978.

[20] M. Biggiogera, S. Fakan, G. Leser, T. E. Martin, and J. Gordon, "Immunoelectron microscopical visualization of ribonucleoproteins in the chromatoid body of mouse spermatids," Molecular Reproduction and Development, vol. 26, no. 2, pp. 150-158, 1990.

[21] F. Moussa, R. Oko, and L. Hermo, "The immunolocalization of small nuclear ribonucleoprotein particles in testicular cells during the cycle of the seminiferous epithelium of the adult rat," Cell and Tissue Research, vol. 278, no. 2, pp. 363-378, 1994.

[22] Y. Onohara, T. Fujiwara, T. Yasukochi, M. Himeno, and S. Yokota, "Localization of mouse vasa homolog protein in chromatoid body and related nuage structures of mammalian spermatogenic cells during spermatogenesis," Histochemistry and Cell Biology, vol. 133, no. 6, pp. 627-639, 2010.

[23] Y. Onohara and S. Yokota, "Expression of DDX25 in nuage components of mammalian spermatogenic cells: immunofluorescence and immunoelectron microscopic study," Histochemistry and Cell Biology, vol. 137, no. 1, pp. 37-51, 2012.

[24] H. Yonetamari, Y. Onohara, and S. Yokota, "Localization of BRUNNOL2 in rat spermatogenic cells as revealed by immunofluorescence and immunoelectron microscopic techniques," Open Journal of Cell Biology, vol. 2, pp. 11-20, 2012.

[25] M. Takebe, Y. Onohara, and S. Yokota, "Expression of MAEL in Nuage and non-Nuage compartments of rat spermatogenic cells and colocalization with DDX4, DDX25 and MIWI," Histochemistry and Cell Biology, vol. 140, no. 2, pp. 169-181, 2013.

[26] S. Yokota and Y. Onohara, "Expression and localization of NANOS1 in spermatogenic cells during spermatogenesis in rat," BioCell, vol. 2, pp. 1-10, 2013.

[27] Y. Clermont, R. Oko, and L. Hermo, "Cell biology of mammalian spermiogenesis," in Cell and Molecular Biology of the Testis, C. Desjardins and L. L. Ewing, Eds., pp. 332-376, Oxford University Press, New York, NY, USA, 1993.

[28] S. Yokota, "Preparation of colloidal gold particles and conjugation of protein A, IgG, F( $\left.\mathrm{ab}^{\prime}\right) 2$, and streptavidin," in Immunoelectron Microscopy: Methods and Protocols., S. D. Schwarzbach and T. Osafune, Eds., pp. 109-119, Springer, New York, NY, USA, 2012.

[29] L. D. Russell, R. A. Ettlin, A. P. S. Hikim, and E. D. Clegg, Histological and Histopathological Evaluation of the Testis, Cache River Press, Clearwater, Fla, USA, 1990.

[30] T. P. Chendrimada, R. I. Gregory, E. Kumaraswamy et al., "TRBP recruits the dicer complex to Ago2 for microRNA processing and gene silencing," Nature, vol. 436, no. 7051, pp. 740$744,2005$.

[31] K. H. Kok, M.-H. J. Ng, Y.-P. Ching, and D.-Y. Jin, "Human TRBP and PACT directly interact with each other and associate 
with dicer to facilitate the production of small interfering RNA," The Journal of Biological Chemistry, vol. 282, no. 24, pp. 1764917657, 2007.

[32] W. Deng and H. Lin, "miwi, a murine homolog of piwi, encodes a cytoplasmic protein essential for spermatogenesis," Developmental Cell, vol. 2, no. 6, pp. 819-830, 2002.

[33] M. A. Carmell, A. Girard, H. J. G. van de Kant et al., "MIWI2 is essential for spermatogenesis and repression of transposons in the mouse male germline," Developmental Cell, vol. 12, no. 4, pp. 503-514, 2007.

[34] L. Russell, "Observations on rat Sertoli ectoplasmic (junctional) specializations in their association with germ cells of the rat testis," Tissue and Cell, vol. 9, no. 3, pp. 475-498, 1977.

[35] B. D. Grove and A. W. Vogl, "Sertoli cell ectoplasmic specializations: a type of actin-associated adhesion junction?" Journal of Cell Science, vol. 93, no. 2, pp. 309-323, 1989.

[36] H. H. N. Yan, D. D. Mruk, W. M. Lee, and C. Y. Cheng, "Ectoplasmic specialization: a friend or a foe of spermatogenesis?" BioEssays, vol. 29, no. 1, pp. 36-48, 2007.

[37] L. Russell and Y. Clermont, "Anchoring device between Sertoli cells and late spermatids in rat seminiferous tubules," Anatomical Record, vol. 185, no. 3, pp. 259-278, 1976.

[38] L. D. Russell, "Spermatid-Sertoli tubulobulbar complexes as devices for elimination of cytoplasm from the head region of late spermatids of the rat," Anatomical Record, vol. 194, no. 2, pp. 233-246, 1979. 

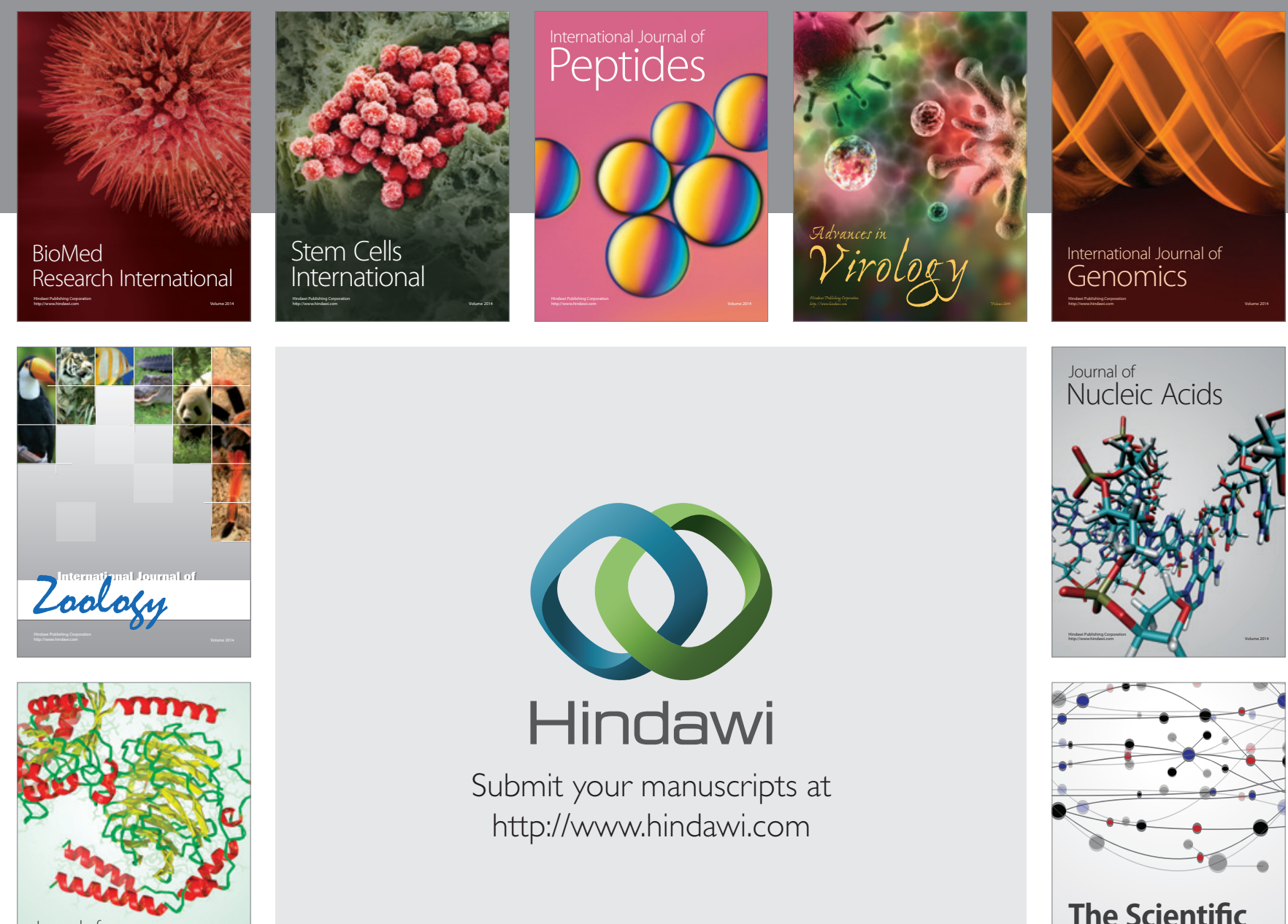

Submit your manuscripts at

http://www.hindawi.com

Journal of
Signal Transduction
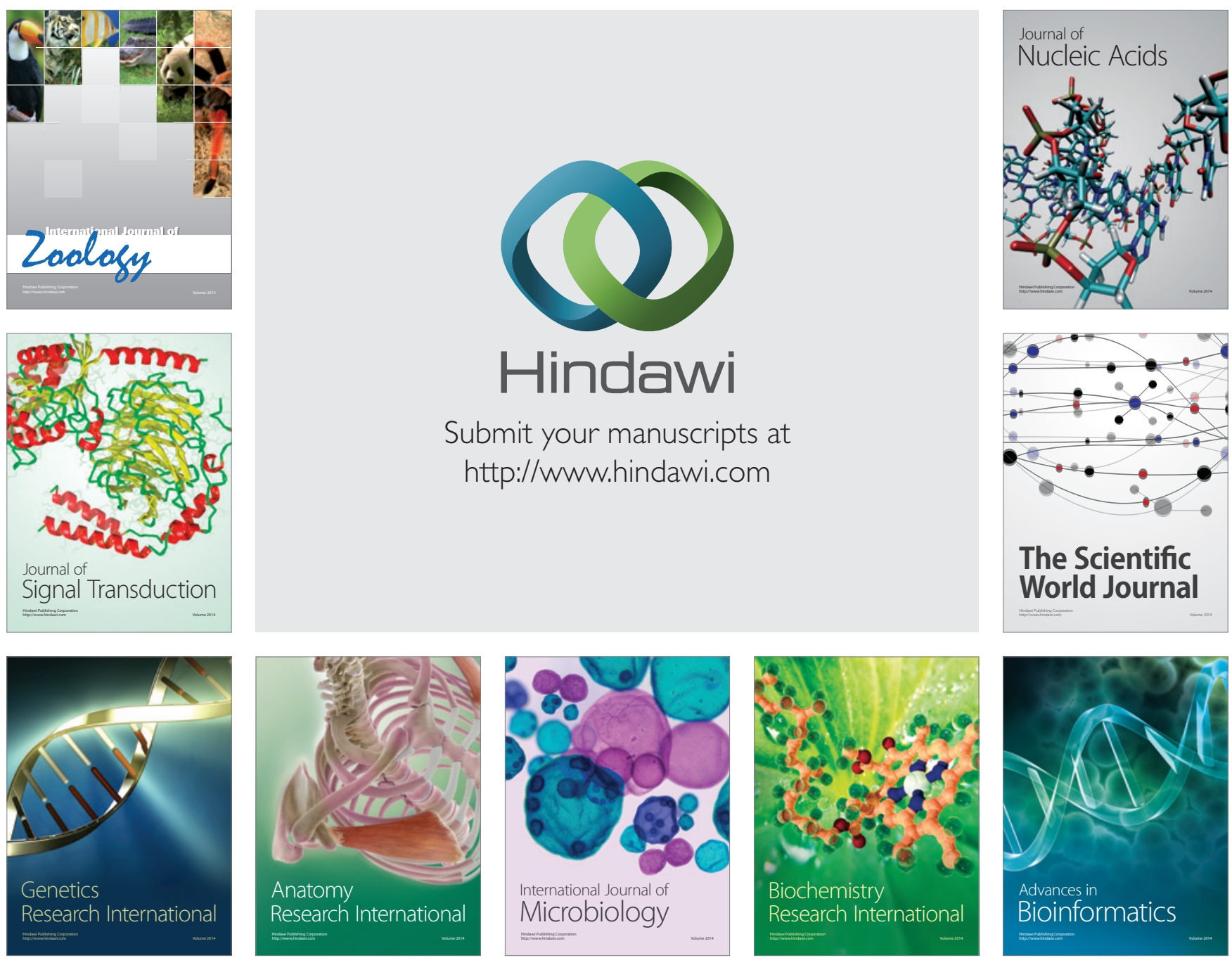

The Scientific World Journal
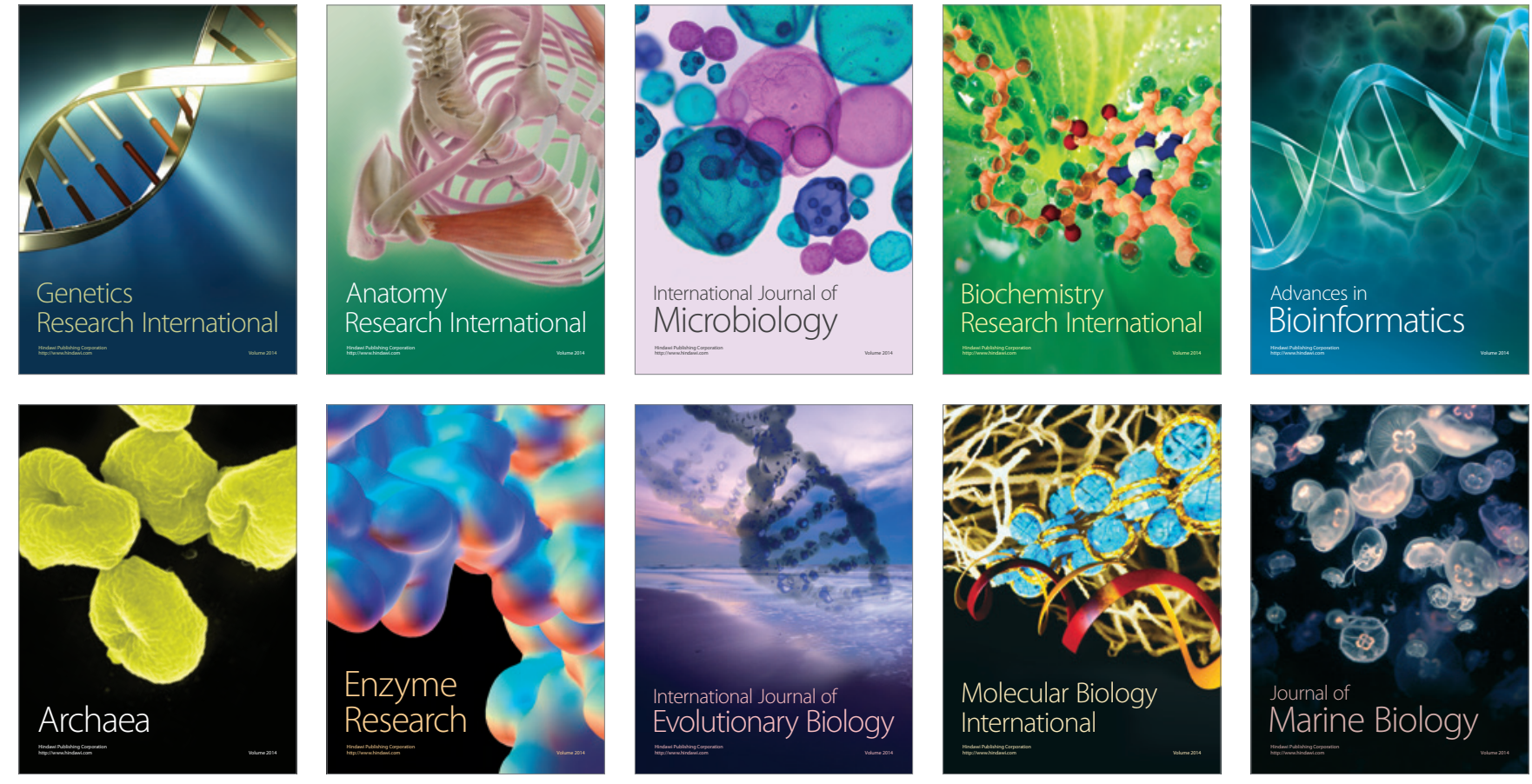